\title{
Análisis bibliométrico de la producción científica de las relaciones públicas a través de las tesis doctorales (2006-2017) y de los programas de doctorado españoles (2017)
}

\section{Joan Cuenca-Fontbona \\ Kathy Matilla}

Universitat Ramon Llull. Facultat de Comunicació i Relacions Internacionals Blanquerna joancf@blanquerna.url.edu

kathyms@blanquerna.url.edu

\section{Marc Compte-Pujol}

Universitat de Vic - Universitat Central de Catalunya. Facultat d'Empresa i Comunicació marc.compte@uvic.cat

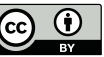

Fecha de presentación: julio de 2018

Fecha de aceptación: mayo de 2019

Fecha de publicación: junio de 2019

Cita recomendada: Cuenca-Fontbona, J.; Matilla, K. y Compte-Pujol, M. (2019). "Análisis bibliométrico de la producción científica de las relaciones públicas a través de las tesis doctorales (2006-2017) y de los programas de doctorado españoles (2017)». Anàlisi. Quaderns de Comunicació i Cultura, 60, 97-117. DOI: <https://doi.org/10.5565/rev/analisi.3166>

\section{Resumen}

Este estudio analiza la producción de tesis doctorales sobre relaciones públicas presentadas en el sistema universitario español durante el periodo 2006-2017. La finalidad ha sido compararla con el mismo estudio del periodo 1965-2005 llevado a cabo por Castillo y Xifra en 2006 y también correlacionar esta producción con los programas de doctorado especializados en relaciones públicas ofertados por las universidades espańolas durante el mismo periodo. La metodología adoptada ha sido el análisis bibliométrico. Así, por un lado, se ha observado que los campos y temas de investigación de la mayoría de las tesis analizadas se refieren al análisis de la práctica profesional de las relaciones públicas y a su dimensión instrumental, resultados similares a los expuestos en la investigación precedente. Por otro lado, el hecho que en estos momentos el sistema universitario español proponga únicamente dos cursos de doctorado con el término «relaciones públicas» en la denominación del título en el curso 2017-2018 y uno más que incluye una línea de investigación de relaciones públicas, condiciona la proliferación de la producción científica de esta disciplina.

Palabras clave: bibliometría; universidad; relaciones públicas en España; tesis doctorales; doctorados 
Resum. Anàlisi bibliomètrica de la producció científica de les relacions públiques a través de les tesis doctorals (2006-2017) i dels programes de doctorat espanyols (2017)

Aquest estudi analitza la producció de tesis doctorals sobre relacions públiques presentades en el sistema universitari espanyol durant el període 2006-2017. La finalitat ha estat comparar-la amb el mateix estudi del període 1965-2005 dut a terme per Castillo i Xifra el 2006 i també correlacionar aquesta producció amb els programes de doctorat especialitzats en relacions públiques oferts per les universitats espanyoles durant el mateix període. La metodologia adoptada ha estat l'anàlisi bibliomètrica. Així, d'una banda, s'ha observat que els camps i temes de recerca de la majoria de les tesis analitzades es refereixen a l'anàlisi de la pràctica professional de les relacions públiques i a la seva dimensió instrumental, resultats similars als exposats en la investigació precedent. D'altra banda, el fet que en aquests moments el sistema universitari espanyol proposi únicament dos cursos de doctorat amb el terme «relacions públiques» en la denominació del títol en el curs 20172018, i un més que inclou una línia d'investigació de relacions públiques condiciona la proliferació de la producció científica d'aquesta disciplina.

Paraules clau: bibliometria; universitat; relacions públiques a Espanya; tesis doctorals; doctorats

Abstract. Bibliometric analysis of scientific production in the field of public relations through doctoral dissertations (2006-2017) and Spanish doctoral programs (2017)

This article analyses the production of doctoral dissertations on public relations read at Spanish universities (2006-2017). The aim is to compare our findings to those of a similar study on the period 1965-2005 carried out by Castillo and Xifra in 2006 and also to correlate this production with doctoral programs specialized in public relations offered by Spanish universities during the same period. The methodology used is bibliometric analysis. On the one hand, it has been observed that the fields and research topics of most of the dissertations analyzed examine the professional practice of public relations and its instrumental dimension, similar to the results presented in the previous research. On the other hand, the fact that the Spanish higher education system offered only two doctoral programs with the term "public relations" in the name of the title in the 2017-2018 academic year and an additional one that included a public relations research line determines the proliferation of this discipline's scientific production.

Keywords: bibliometrics; university; public relations in Spain; doctoral dissertations; doctorates

\section{Introducción}

En 2006, Castillo y Xifra publicaron en el número 34 de la revista Anàlisi. Quaderns de Comunicació i Cultura el primer estudio bibliométrico (González de Dios et al., 1997; Maltras-Barba, 2003) sobre las tesis doctorales presentadas en las universidades espańolas, públicas y privadas, entre 1965 -fecha de defensa de la primera tesis doctoral especializada en relaciones públicas - y 2005. Este artículo pretende ofrecer una extensión y comparación del estudio de este periodo sobre la investigación doctoral en relaciones públicas en las universidades españolas realizada, en esta ocasión, entre 2006 y 2017. 
El uso de indicadores bibliométricos para estudiar la actividad investigadora se basa en que las publicaciones científicas son resultado esencial de dicha actividad, y proporcionan información sobre los resultados del proceso investigador, su volumen, evolución, visibilidad y estructura. Así, permiten valorar la actividad científica, e influencia (o impacto) tanto del trabajo como de las fuentes. Los estudios bibliométricos, en conjunto con otros indicadores, permiten la cuantificación de la ciencia en forma objetiva, y se potencian con la explosión actual del conocimiento y su recopilación en las bases bibliográficas de datos. (Camps, 2008: 74)

En el análisis comparativo y su contextualización se han tenido en cuenta algunas cuestiones históricas: la evolución de los estudios universitarios que incluían las relaciones públicas, los programas de doctorado y las publicaciones científicas relacionadas con esta materia.

\subsection{Periodo $1965-2005$}

Respecto a la investigación de Castillo y Xifra (2006), durante el régimen dictatorial franquista (1939-1975) que alcanzó los primeros diez años del periodo analizado (1965-1975), la universidad espańola se caracterizó por una gran pobreza teórica y ausencia de rigor académico y científico. Los estudios universitarios especializados en relaciones públicas no cristalizaron hasta que, en 1974, las universidades Complutense de Madrid y Autónoma de Barcelona ofertaran las primeras licenciaturas de Publicidad y Relaciones Públicas (ibidem: 146-147).

Entre 1985 y 1995 se reformaron los estudios de Ciencias de la Información, que dieron paso a tres licenciaturas: Periodismo, Comunicación Audiovisual y Publicidad y Relaciones Públicas (ibidem: 150). En el año 2000, el número de universidades españolas que contaban con licenciaturas en Publicidad y Relaciones Públicas ya ascendía a 19 (Tilson y Pérez, 2003: 151) y seis años después, en 2006, el número ya era de 32 (ibidem: 149).

Los doctorados especializados en relaciones públicas fueron inexistentes entre 1974 y 2003, hasta que la Universidad de Málaga ofertó un primer programa de Doctorado en Comunicación Organizacional, que incluyó una línea de investigación en publicidad y relaciones públicas. Sin embargo, las dos primeras tesis sobre relaciones públicas defendidas en España datan de 1965 y 1966. Ambas se presentaron en la Facultad de Ciencias Políticas, Económicas y Comerciales de la desaparecida Universidad de Madrid (ibidem: 150-151).

La primera publicación científica española sobre comunicación social fue la revista Anàlisi. Quaderns de Comunicació i Cultura, de la Universidad Autónoma de Barcelona en 1980, ya en plena democracia, y no será hasta 2003 que la Universidad de Sevilla publique el primer número de la primera revista especializada en relaciones públicas: Laurea (ibidem: 146). 


\subsection{Periodo $2006-2017$}

El segundo periodo analizado, objeto de esta investigación, coincide temporalmente con la implantación y despliegue en España del Espacio Europeo de Educación Superior (EEES) y la creación de las agencias de evaluación de la calidad científica (Agencia Nacional de Evaluación de la Calidad y Acreditación [ANECA], Comisión Nacional Evaluadora de la Actividad Investigadora [CNEAI]), constituyendo el logro de grado de doctor una titulación vital, y la consiguiente calidad de la productividad investigadora en el indicador cuantitativo clave para la promoción profesional del profesorado universitario.

El número de grados en Publicidad y Relaciones Públicas —o equivalentes- de la oferta universitaria española era de 33 en el curso académico 2014-2015 (Perlado y Saavedra, 2017: 104). Y el primer y único doctorado con contenidos específicos en relaciones públicas existente en España aparece en 2006 en la Universidad de Málaga (Castillo et al., 2013: 106), en un contexto general en el que Cuenca et al. (2017: 40) señalan que ya coexistían 829 másters universitarios.

En el curso 2012-2013 Castillo et al. (2013: 105) identificaron 32 titulaciones de máster universitario (oficial) en relaciones públicas, que representaban un total de 1.095 plazas disponibles. Paulatinamente, a las relaciones públicas se van ańadiendo otras denominaciones que dan cuenta del desplazamiento de la disciplina en beneficio de otros enfoques profesionales - como la comunicación corporativa o la dirección de comunicación- $y$, así, un año después, en el curso académico 2013-2014, Matilla et al. (2014) comprobaron que el número de másters universitarios especializados en relaciones públicas y/o comunicación corporativa adaptados al EEES había disminuido a 23 y, en el curso 20142015, el total de los másters universitarios relacionados con la dirección de comunicación era de 22 (Perlado y Saavedra, 2017: 88). Dos cursos académicos después, en 2016-2017, Cuenca et al. (2017: 47) identifican tan solo 9 másters oficiales en el sistema universitario español, en los que figuraban los conceptos «relaciones públicas», "comunicación corporativa» o "comunicación» en sus nomenclaturas, siendo 4 de ellos de nueva creación, ofertados por las universidades Carlos III de Madrid, La Rioja, Rey Juan Carlos y Europea del Atlántico.

Los últimos datos provisionales del Ministerio de Educación, Cultura y Deporte del Estado Español (2017) muestran que en el curso 2016-2017 la cifra de nuevas incorporaciones en los másters universitarios fue de 13.702 $(0,87 \%)$ estudiantes. A nivel de doctorado, hubo un incremento de 10.851 estudiantes (19,5\%), en ambos casos con respecto al curso anterior. No se han identificado datos específicos sobre el número de estudiantes en el ámbito de las relaciones públicas.

\section{Marco teórico}

Centrándonos en nuestro objeto de estudio, a continuación expondremos una panorámica sobre el estado del arte de las tesis doctorales en el Estado Español. 


\subsection{Las tesis doctorales en España}

La literatura especializada sobre la producción española de tesis doctorales es abundante. En el periodo 1997-2008, Fuentes y Arguimbau (2010: 66) identificaron 18 áreas temáticas o campos científicos, entre los que aparecen las relaciones públicas analizadas por Castillo y Xifra en 2006.

En el contexto de grandes cambios que está experimentando el sistema universitario español en la última década, con la convergencia al EEES han proliferado los estudios sobre la productividad científica, entre los que destacan, por ejemplo, los estudios bibliométricos sobre tesis doctorales dirigidas en España. Para Moreno y Moreno (2016: s.f.), las tesis doctorales constituyen un trabajo inédito que aporta nuevos conocimientos al campo científico y son un excelente referente para conocer la estructura social de la investigación en la universidad, por lo que se han constituido en sí mismas en un objeto de investigación en el que se definen las líneas, tendencias y potencialidades de la investigación universitaria.

Fuentes y Arguimbau (2010: 64) afirman que, antes de la expansión de Internet, las tesis doctorales formaban parte de la literatura "gris", ya que su difusión y acceso estaban muy limitados (Merlo y Sorli, 2002; Miguel, 2000; Moralejo, 2000; Orera, 2003; Agudelo et al., 2003) y advierten que en la producción de tesis doctorales en España, en el periodo 1997-2008, se produce una transformación condicionada por el proceso de adaptación al EEES, el impacto de las Tecnologías de la Información y de las Comunicaciones (TIC) y, por último, el movimiento orientado al acceso abierto (open access) de los documentos científicos, que incrementan su difusión, su identificación y su visibilidad. Para Peñafiel et al. (2016: 793), Internet ha traído numerosos cambios, que están cada día más consolidados en el terreno de la difusión de la producción científica. La comunicación y su estudio no pueden mantenerse al margen del efecto disruptivo ocasionado por esas transformaciones (Tato et al., 2014: 10). El fenómeno de la digitalización ha aumentado el número de investigaciones y tesis doctorales españolas registradas en las bases de datos TESEO (García Leiva, 2011).

Para comprender el panorama de las tesis doctorales especializadas en relaciones públicas en España resulta ilustrativa la contextualización que proporciona la literatura internacional sobre otros países. En 2007, Watson (2008a) llevó a cabo un estudio Delphi con académicos y profesionales, de ámbito internacional, para identificar cuáles serían los temas de investigación prioritarios en relaciones públicas en los siguientes diez años. Para ello, se basó en estudios similares anteriores desarrollados por McElreath (1980, 1989) —Estados Unidos de América-; White y Blamphin (1994), McElreath y Blamphin (1994) —Reino Unido—; Synnott y McKie (1997) — 13 países de Asia-Pacífico-; Wakefield (2000), Van Ruler et al. (2004) - 22 y 25 países de Europa, respectivamente-; Raupp y Van Ruler (2006) —Holanda y Alemania-; y Boynton (2006), y concluyó que: 
The role of public relations in the strategic operation of organisations, and the creation of value by public relations through social capital and relationships, were ranked as the top two priorities. Some outcomes were comparable with earlier studies; for instance, evaluation of public relations programmes ranked third in this study and was also among the leaders in the most recent similar study. Only 'management of relationships' was wholly new. (Watson, 2008a: p. n.e.)

Watson, a partir de su estudio, también subrayó que:

It can be repeated with similar methods and samples so that there is a rolling benchmark of the issues and topics that contribute to developing the public relations body of knowledge (Broom, 2006). One of the drivers behind this research has been the increasingly demanding processes of bidding for funding of research that call for relevance and potential for implementation. By identifying these priorities, it is hoped that they will give legitimacy to bids from public relations researchers, who can demonstrate them as an international academic/practitioner benchmark in support of their proposals. (Watson, 2008a: p. n.e.)

Según Míguez et al. (2016: 14), la investigación aplicada es la preferida por los investigadores de relaciones públicas que eligen el Journal of Public Relations Research para difundir sus trabajos (57\%). El resto se reparte de forma prácticamente equitativa entre investigación básica (23\%) e introspectiva $(20 \%)$. La investigación aplicada es mayoritaria en las diez primeras revistas por factor de impacto especializadas en comunicación en el año 2008, seleccionadas en base a la Ley Bradford, en seis de las cuales se publicaron temas de relaciones públicas. La autoría masculina fue superior a la femenina en el periodo analizado (Castillo-Esparcia et al., 2012: p.n.e., Cuadro 8).

\subsection{Los programas de doctorado en España}

Castillo et al. (2013) afirman que la totalidad de la oferta española de programas de doctorado de todas las disciplinas académicas ascendía a 57 en 2012. La estructura de la nueva oferta de estudios de postgrado surgida del RD 99/2011, de 28 de enero, que regulaba las enseñanzas oficiales de doctorado, provocará cambios profundos, debido a que:

los requisitos establecidos por la normativa han producido una reducción considerable de la oferta de doctorado. Esta oferta se ha estructurado bajo dos modelos. Un modelo horizontal con la creación de programas de doctorado en comunicación interuniversitario y un modelo vertical con programas de doctorado intrauniversitarios cuya denominación más usual son doctorados en ciencias sociales que incluyen una o varias líneas de investigación en comunicación y una amplitud y generalización de las líneas temáticas. Igualmente se tiende a una confluencia de áreas de conocimiento bajo las cuales poder ofertar dichos programas y hay una reducción drástica de la oferta de 
plazas, así como una reducción del profesorado implicado debido a la exigencia de requisitos previos (sexenios, publicaciones, grupos de investigación...). (Castillo et al., 2016: 703)

En base a ello, tres años después, en el curso 2015-2016 la oferta total de programas de doctorado en España se había reducido un 60\%, de 57 a 24 programas (Castillo et al., 2016: 708), observándose una confluencia hacia la interdisciplinariedad de los centros responsables, hecho que les ha permitido seguir manteniendo viva la oferta de postgrado (ibidem: 715).

Actualmente, las universidades españolas que han diseñado un plan estratégico global, alineado con las prioridades europeas, son aún muy escasas. Los doctorados españoles siguen focalizándose casi en exclusividad en perfiles de futuros académicos, una salida profesional con magras perspectivas de éxito debido a los escasos puestos de trabajo que se prevé se generarán en territorio europeo en las próximas décadas (Haug, 2015: 35). Por consiguiente, queda aún mucho camino por recorrer, ya que la reforma y la modernización de los doctorados - junto con el fortalecimiento de los másters oficiales, a los que están indisolublemente unidos - se perfila como prioritaria en el sistema universitario español frente al objetivo de plena equiparación de calidad con algunos de sus homólogos europeos (ibidem: 35 ).

En el campo específico de las relaciones públicas, la Universidad de Málaga fue la primera en ofertar, en 2006, un doctorado que incluía la materia: el Doctorado en Comunicación Organizacional (Castillo y Xifra, 2006: 150), que se mantuvo inalterable hasta el año 2013, cuando pasó a ser el máster interuniversitario. Se destaca el hecho de que la escasez de programas de doctorado especializados en relaciones públicas no es patrimonio exclusivo del sistema universitario español: Botan y Hazleton ya habían denunciado la misma situación a nivel internacional en 2006 (ibidem: 151).

\section{Metodología}

La metodología adoptada ha sido la misma que se utilizó en el estudio anterior de Castillo y Xifra (2006), consistente en un análisis bibliométrico aplicado al universo de las 38 tesis doctorales presentadas en España entre 2006 y 2017 cuyo objeto de estudio eran las relaciones públicas. No se han planteado innovaciones metodológicas ni incorporado nuevos puntos de vista a efectos de favorecer al máximo el análisis comparativo entre ambos periodos analizados.

Este análisis sobre el ámbito, la estructura y las características de la investigación abarca los títulos de las tesis, las fechas de sus presentaciones y las universidades en las que han tenido lugar. Para determinar la muestra se seleccionaron y analizaron la totalidad de las 38 tesis doctorales presentadas en las universidades públicas y privadas de Espańa desde 2006 hasta 2017, en cuyo "título», «resumen» y/o "palabras clave o descriptor» aparecía el término relaciones públicas. Nos interesaron las tesis doctorales que los doctorandos habían 
considerado que debían etiquetarse con dicho código, ya que ello implicaba un posicionamiento claro por parte del investigador. No se contemplaron las tesis en cuyo resumen o bibliografía se hacía referencia al concepto «relaciones públicas» o bien se manifestaba haber entrevistado a profesionales de las relaciones públicas; pero no se trataba ningún aspecto de esta disciplina.

Siguiendo a Castillo y Xifra (2006), la investigación se llevó a cabo mediante la revisión de dos bases de datos en línea: (1) Base de Datos de Tesis Doctorales TESEO del Consejo de Coordinación Universitaria del Ministerio de Educación, Cultura y Deporte de España (<https://www.educacion.gob.es/ teseo/irGestionarConsulta.do $>$ ). La información de la ficha de la tesis incluida en la Base de Datos de Tesis Doctorales TESEO, además de los datos del autor y del tribunal, incluye un resumen detallado de la misma y los descriptores fundamentales; y (2) la base de datos del Servei de Tesis Doctorals en Xarxa-TDX gestionado por el Consorcio de Servicios Universitarios de Cataluña (CSUC) (<http://www.tesisenred.net $>$ ), que contiene, en formato digital, tesis doctorales leídas en las universidades de Cataluña y en otras comunidades autónomas. En esta base de datos, patrocinada por la Generalitat de Cataluña, la información que se ofrece de cada uno de los registros de las tesis es la siguiente: los datos del autor, el resumen de la tesis y las palabras clave de la investigación. En este sentido, es importante señalar que la inclusión de las tesis doctorales en ambas bases de datos es voluntaria, por lo que cabe la posibilidad que la muestra analizada no se corresponda exactamente con el universo.

La consulta sistemática de las 38 tesis doctorales presentadas en España entre 2006 y 2017 cuyo objeto de estudio eran las relaciones públicas se desarrolló entre el 2 y el 6 de julio de 2018 y con la intervención no participante suplementaria de dos investigadores independientes que supervisaron generosamente la totalidad del trabajo de campo desarrollado para garantizar, con la máxima objetividad y precisión, la validez científica de los datos.

Se está conforme con Castillo y Xifra (2006) respecto a que las tesis doctorales deberían ser el primer indicador del estado evolutivo del desarrollo teórico de las relaciones públicas, así como constituir el origen de futuros artículos en revistas científicas. Así pues, con respecto a la sistematización de los contenidos, la codificación se estableció siguiendo la misma metodología empleada por Castillo y Xifra (2006), que contemplaba 3 categorías y 38 subcategorías basadas en la categorización elaborada por Sallot et al. (2003) que, a su vez, estaban fundamentadas en el análisis de Ferguson (1984) sobre el estado de la elaboración teórica de las relaciones públicas. Así, las tres variables que se tuvieron en cuenta fueron: (a) tesis doctorales introspectivas, es decir, centradas en desarrollar nuevas teorías acerca de las relaciones públicas; (b) tesis sobre aplicación y práctica de las relaciones públicas, y (c) tesis relacionadas con el desarrollo teórico de las relaciones públicas.

Después de codificar las tesis, según lo especificado, y una vez eliminadas las categorías que quedaron desiertas, las tesis denominadas introspectivas incluyeron las siguientes subcategorías: 
- pedagogía/educación en relaciones públicas

- ética y responsabilidad social

- historia de las relaciones públicas

- profesión de relaciones públicas

- práctica internacional de las relaciones públicas

Las tesis que trataban sobre la práctica/aplicación de las relaciones públicas se ordenaron de la siguiente forma:

- dirección/toma de decisiones/resolución de conflictos

- implantación de programas y campañas

- comunicación organizacional

- gestión de conflictos potenciales y temas sociales

- nuevas tecnologías de la comunicación

- cuestiones legales

- comunicación de crisis

- gestión de la imagen/reputación/impresión

- ética (en la práctica)

- cuestiones y metodologías de investigación aplicada

Por último, la taxonomía resultante de las tesis sobre el desarrollo teórico de las relaciones públicas fue:

- teoría de la excelencia/comunicación simétrica/modelos de Grunig

- captación de fondos

- relaciones con los públicos

- comunicación organizacional

- teoría/modelos de la función de relaciones públicas

Siguiendo a Castillo y Xifra (2006), las unidades de análisis registradas fueron el título, el idioma utilizado, el resumen de las tesis obtenidas en las bases de datos, TESEO y TDX, las palabras clave en TDX, y los descriptores en TESEO. Se registraron los nombres de los autores, las universidades donde se presentaron y el año de la defensa.

Por otro lado, para identificar los programas de doctorado objeto de este estudio se utilizó un muestreo no probabilístico por propósito en base al universo de titulaciones de doctorado presentes en dos buscadores distintos: (a) el buscador web de la Agencia Nacional de Evaluación de la Calidad y Acreditación (ANECA), titulado "¿Qué estudiar y dónde?» (<http://srv.aneca.es/ ListadoTitulos/busqueda-titulaciones $>$ ) y (b) el buscador de títulos oficiales del Ministerio de Educación, Cultura y Deporte (<https://www.educacion. gob.es/notasdecorte/compBdDo $>$ ). En ambos casos se eligieron los títulos de doctorados universitarios que se imparten en España en el curso 2016-2017 y que ostentan los términos «relaciones públicas» en su denominación. Una vez identificada la oferta, se contrastó mediante la visita a sus webs oficiales, para certificar que actualmente estos programas se continúan ofertando. 


\section{Resultados}

\subsection{Tesis doctorales sobre Relaciones Públicas (2006-2017)}

Los principales resultados se detallan a continuación en las dos primeras columnas de la Tabla 1. Las columnas tercera y cuarta corresponden a los del estudio anterior (1965-2005) de Castillo y Xifra (2006). Se observa que en esta tabla aparecen nuevas subcategorías que en el anterior estudio no salían. Esto es porque en el presente análisis hay tesis doctorales que pertenecen a subcategorías que, en el estudio de 2006, quedaron desiertas.

Tabla 1. Categorización de las tesis españolas sobre RP (1965-2005 vs 2006-2017)

\begin{tabular}{|c|c|c|c|c|}
\hline Categorías & $\begin{array}{l}\text { N. }{ }^{\circ} \text { tesis } \\
2006-2017\end{array}$ & $\begin{array}{c}\% \text { tesis } \\
2006-2017\end{array}$ & $\begin{array}{l}\text { N. }{ }^{\circ} \text { tesis } \\
1965-2005\end{array}$ & $\begin{array}{l}\% \text { tesis } \\
1965-2005\end{array}$ \\
\hline Introspectiva & 5 & 13,15 & 12 & 18,46 \\
\hline Profesión de Relaciones Públicas & 2 & 5,26 & 4 & 6,15 \\
\hline Pedagogía / Educación en Relaciones Públicas & 1 & 2,63 & 2 & 3,08 \\
\hline Historia de las Relaciones Públicas & 1 & 2,63 & 1 & 1,54 \\
\hline $\begin{array}{l}\text { Práctica internacional de las Relaciones } \\
\text { Públicas }\end{array}$ & 0 & 0,00 & 2 & 3,08 \\
\hline Ética y responsabilidad social & 1 & 2,63 & 3 & 4,61 \\
\hline Práctica/aplicación de las Relaciones Públicas & 25 & 65,77 & 47 & 72,31 \\
\hline Implantación de programas y campañas & 10 & 26,31 & 19 & 29,23 \\
\hline $\begin{array}{l}\text { Gestión de conflictos potenciales y temas } \\
\text { sociales }\end{array}$ & 0 & 0,00 & 2 & 3,08 \\
\hline $\begin{array}{l}\text { Cuestiones y metodologías de investigación } \\
\text { aplicada }\end{array}$ & 3 & 7,89 & 0 & 0,00 \\
\hline Comunicación organizacional & 2 & 5,26 & 7 & 10,77 \\
\hline Comunicación de crisis & 0 & 0,00 & 2 & 3,08 \\
\hline $\begin{array}{l}\text { Dirección / Toma de decisiones / Resolución de } \\
\text { conflictos }\end{array}$ & 0 & 0,00 & 4 & 6,15 \\
\hline Nuevas tecnologías de la comunicación & 7 & 18,42 & 6 & 9,23 \\
\hline Cuestiones legales & 0 & 0,00 & 1 & 1,54 \\
\hline Ética (en la práctica) & 2 & 5,26 & 0 & 0,00 \\
\hline Gestión de la imagen / Reputación / Impresión & 1 & 2,63 & 6 & 9,23 \\
\hline Desarrollo teórico de las Relaciones Públicas & 8 & 21,04 & 2 & 3,08 \\
\hline $\begin{array}{l}\text { Teoría de la excelencia / Comunicación } \\
\text { simétrica / Modelos de Grunig }\end{array}$ & 1 & 2,63 & 1 & 1,54 \\
\hline Relaciones con los públicos & 5 & 13,15 & 0 & 0,00 \\
\hline Comunicación organizacional & 1 & 2,63 & 0 & 0,00 \\
\hline $\begin{array}{l}\text { Teoría/modelos de la función de Relaciones } \\
\text { Públicas }\end{array}$ & 1 & 2,63 & 0 & 0,00 \\
\hline Fundraising (captación de fondos) & 0 & 0,00 & 1 & 1,54 \\
\hline Sin categorizar (desacuerdo entre codificadores) & 0 & 0,00 & 4 & 6,15 \\
\hline TOTALES & 38 & 100,00 & 65 & 100,00 \\
\hline
\end{tabular}

Fuente: elaboración propia, 2006-2017. Castillo y Xifra (2006: 152), 1965-2005. 
La categoría acerca de la práctica y aplicación profesional de las relaciones públicas es la categoría que contiene la mayor producción científica, con 25 trabajos $(65,77 \%)$, mientras que la suma de los trabajos de las categorías de carácter introspectivo y acerca del desarrollo teórico de las relaciones públicas se eleva a 13 tesis $(34,19 \%)$. En especial, destacan las subcategorías sobre la implantación de programas y campañas, con 10 tesis (26,31\%), y sobre nuevas tecnologías de la comunicación, con 7 tesis $(18,42 \%)$. Entre las dos representan cerca de la mitad de la producción total (44,73\%).

El resto de las subcategorías temáticas aparecen muy polarizadas, con diversidad de materias tratadas. La práctica internacional de las relaciones públicas, la gestión de conflictos potenciales y temas sociales, la comunicación de crisis, la resolución de conflictos, las cuestiones legales y la captación de fondos no han sido objeto de ningún estudio doctoral en el periodo analizado. Sí, en cambio, durante este periodo, las cuestiones y metodologías de investigación aplicada, la ética (en la práctica), la comunicación organizacional, la teoría/modelos de la función de relaciones públicas y, sobresaliendo con 5 tesis, la subcategoría acerca de las relaciones con los públicos.

Tabla 2. Tesis doctorales españolas sobre Relaciones Públicas (2006-2017)

\begin{tabular}{|c|c|c|c|}
\hline $\begin{array}{l}\text { BAPTISTA, Joyce } \\
\text { Isabel }\end{array}$ & 2016 & $\begin{array}{l}\text { Diplomacia pública de las redes de ciudades } \\
\text { europeas. Análisis de la calidad de las } \\
\text { relaciones internas }\end{array}$ & $\begin{array}{l}\text { Universidad de } \\
\text { Navarra }\end{array}$ \\
\hline $\begin{array}{l}\text { BARQUERO, Carmen } \\
\text { Fátima }\end{array}$ & 2011 & $\begin{array}{l}\text { La implementación estratégica de las } \\
\text { franquicias. Aplicación de las relaciones } \\
\text { públicas en la franquicia corporativa y el } \\
\text { franquiciado }\end{array}$ & $\begin{array}{l}\text { Universidad } \\
\text { Camilo José } \\
\text { Cela }\end{array}$ \\
\hline $\begin{array}{l}\text { BARQUERO, José } \\
\text { Daniel }\end{array}$ & 2011 & $\begin{array}{l}\text { Convergencia del marketing y de las relaciones } \\
\text { públicas con las nuevas políticas estratégicas } \\
\text { de la alta administración y dirección de } \\
\text { empresas en la persuasión de los públicos y } \\
\text { mercados }\end{array}$ & $\begin{array}{l}\text { Universidad } \\
\text { Camilo José } \\
\text { Cela }\end{array}$ \\
\hline BEAMUD, Agustín & 2013 & $\begin{array}{l}\text { Uso de las relaciones públicas e integración de } \\
\text { esta herramienta de comunicación en planes } \\
\text { estratégicos de las asociaciones empresariales } \\
\text { valencianas }\end{array}$ & $\begin{array}{l}\text { Universidad } \\
\text { Cardenal } \\
\text { Herrera-CEU }\end{array}$ \\
\hline BLANCO, Félix & 2009 & $\begin{array}{l}\text { Prensa y relaciones públicas en el ámbito local: } \\
\text { análisis de la gestión de la comunicación de tres } \\
\text { organizaciones de Vigo (1976-2000) }\end{array}$ & $\begin{array}{l}\text { Universidad de } \\
\text { Vigo }\end{array}$ \\
\hline COMPTE, Marc & 2017 & $\begin{array}{l}\text { La estrategia de comunicación del patrimonio } \\
\text { desde la comunicación corporativa y las } \\
\text { relaciones públicas. Análisis de un caso: el } \\
\text { patrimonio de la humanidad de la UNESCO en } \\
\text { España }\end{array}$ & $\begin{array}{l}\text { Universidad } \\
\text { Ramon Llull }\end{array}$ \\
\hline CUENCA, Joan & 2011 & $\begin{array}{l}\text { El análisis de la calidad de las relaciones en el } \\
\text { ámbito de las relaciones públicas. Aplicación de } \\
\text { un modelo global de auditoría de relaciones } \\
\text { públicas a la sociedad municipal 22@ del } \\
\text { Ayuntamiento de Barcelona }\end{array}$ & $\begin{array}{l}\text { Universidad } \\
\text { Ramon Llull }\end{array}$ \\
\hline
\end{tabular}




\begin{tabular}{|c|c|c|c|}
\hline ESTANYOL, Elisenda & 2014 & $\begin{array}{l}\text { Percepció i gestió de la creativitat en les } \\
\text { empreses consultores de comunicació i } \\
\text { relacions públiques }\end{array}$ & $\begin{array}{l}\text { Universidad } \\
\text { Autónoma de } \\
\text { Barcelona }\end{array}$ \\
\hline $\begin{array}{l}\text { Franco, Carlos } \\
\text { Iván }\end{array}$ & 2016 & $\begin{array}{l}\text { Modelo de protocolo y ceremonial español } \\
\text { adaptado en el contexto del Estado Mexicano }\end{array}$ & $\begin{array}{l}\text { Universidad } \\
\text { Complutense } \\
\text { de Madrid }\end{array}$ \\
\hline GARcíA, Carlos & 2015 & $\begin{array}{l}\text { Relaciones públicas. } 2.0 \text { : la personalización de } \\
\text { los mensajes en redes sociales como elemento } \\
\text { de fidelización }\end{array}$ & $\begin{array}{l}\text { Universidad de } \\
\text { Málaga }\end{array}$ \\
\hline GARCÍA, Ma Dolores & 2014 & $\begin{array}{l}\text { Comunicación y casa real. La organización de } \\
\text { actos como estrategia de relaciones públicas de } \\
\text { la casa de S.M. el Rey }\end{array}$ & $\begin{array}{l}\text { Universidad de } \\
\text { Málaga }\end{array}$ \\
\hline $\begin{array}{l}\text { GodoY, Francisco } \\
\text { Javier }\end{array}$ & 2017 & $\begin{array}{l}\text { Web } 2.0 \text { y comunicación. Análisis transversal de } \\
\text { la web participativa en el ámbito de las } \\
\text { relaciones públicas }\end{array}$ & $\begin{array}{l}\text { Universidad de } \\
\text { Málaga }\end{array}$ \\
\hline $\begin{array}{l}\text { GonZÁLEZ, Enrique } \\
\text { Manuel }\end{array}$ & 2009 & $\begin{array}{l}\text { La evaluación del impacto de las campañas de } \\
\text { relaciones públicas. Un estudio de su práctica } \\
\text { real en el sector español de la automoción }\end{array}$ & $\begin{array}{l}\text { Universidad } \\
\text { Rey Juan } \\
\text { Carlos }\end{array}$ \\
\hline $\begin{array}{l}\text { LORENZO, } \\
\text { Francisco }\end{array}$ & 2013 & $\begin{array}{l}\text { Las relaciones públicas en la estrategia de } \\
\text { comunicación de la guardia civil de Alicante: } \\
\text { aplicación de modelos conductuales }\end{array}$ & $\begin{array}{l}\text { Universidad de } \\
\text { Alicante }\end{array}$ \\
\hline LoZANO, Natalia & 2015 & $\begin{array}{l}\text { Critical PR in fool communication and social } \\
\text { media. The case of European food safety and } \\
\text { promotion authorities }\end{array}$ & $\begin{array}{l}\text { Universidad } \\
\text { Rovira i Virgili }\end{array}$ \\
\hline MAMIC, Lilia Ivana & 2014 & $\begin{array}{l}\text { Communication of larga corporation on Twitter: } \\
\text { public relations' models, interactivity patterns } \\
\text { and tools, and the impact of corporate social } \\
\text { responsibility contents on their publics }\end{array}$ & $\begin{array}{l}\text { Universidad } \\
\text { Rey Juan } \\
\text { Carlos }\end{array}$ \\
\hline MARCA, Guillem & 2011 & $\begin{array}{l}\text { La evaluación en los modelos de planificación } \\
\text { estratégica de las relaciones públicas. Análisis } \\
\text { comparativo del uso de la evaluación de la } \\
\text { comunicación en las redes hospitalarias de los } \\
\text { modelos sanitarios de España, el Reino Unido y } \\
\text { Estados Unidos }\end{array}$ & $\begin{array}{l}\text { Universidad } \\
\text { Rovira i Virgili }\end{array}$ \\
\hline MATILLA, Kathy & 2007 & $\begin{array}{l}\text { Aportaciones para un modelo global de } \\
\text { planificación estratégica en relaciones públicas } \\
\text { y comunicación integral. Análisis de un caso }\end{array}$ & $\begin{array}{l}\text { Universidad } \\
\text { Ramon Llull }\end{array}$ \\
\hline OLIVEIRA, Andrea & 2012 & $\begin{array}{l}\text { Los públicos en la etapa de investigación de la } \\
\text { planificación estratégica de las relaciones } \\
\text { públicas. Estudio de los públicos en las } \\
\text { empresas productoras, distribuidoras y } \\
\text { comercializadoras de gas natural y energía } \\
\text { eléctrica en España }\end{array}$ & $\begin{array}{l}\text { Universidad } \\
\text { Rovira i Virgili }\end{array}$ \\
\hline ORDEIX, Enric & 2014 & $\begin{array}{l}\text { Constructing the social personality in } \\
\text { organizations: essentials of managing the social } \\
\text { commitment according to the parameters } \\
\text { reached by the study "excellence in public } \\
\text { relations and communication management» }\end{array}$ & $\begin{array}{l}\text { Universidad } \\
\text { Ramon Llull }\end{array}$ \\
\hline PALAU, Ramon & 2006 & $\begin{array}{l}\text { Relacions públiques i responsabilitat social } \\
\text { corporativa a les cadenes hoteleres catalanes }\end{array}$ & $\begin{array}{l}\text { Universidad } \\
\text { Autónoma de } \\
\text { Barcelona }\end{array}$ \\
\hline
\end{tabular}




\begin{tabular}{|c|c|c|c|}
\hline $\begin{array}{l}\text { PASCUAL, María } \\
\text { Cristina }\end{array}$ & 2015 & $\begin{array}{l}\text { Las relaciones públicas de los despachos de } \\
\text { abogados: análisis de la comunicación de los } \\
\text { bufetes en España }\end{array}$ & $\begin{array}{l}\text { Universidad } \\
\text { Complutense } \\
\text { de Madrid }\end{array}$ \\
\hline PÉREZ, Antonia & 2017 & $\begin{array}{l}\text { Modelos y técnicas de relaciones públicas } \\
\text { aplicables a las agencias de empleo y desarrollo } \\
\text { local en el marco de la gestión y promoción } \\
\text { turística en la provincia de A Coruña }\end{array}$ & $\begin{array}{l}\text { Universidad de } \\
\text { A Coruña }\end{array}$ \\
\hline $\begin{array}{l}\text { POLO, Juan } \\
\text { Francisco }\end{array}$ & 2009 & $\begin{array}{l}\text { La responsabilidad social corporativa de las } \\
\text { grandes empresas españolas con presencia en } \\
\text { América Latina: modelos de actuación }\end{array}$ & $\begin{array}{l}\text { Universidad } \\
\text { Complutense } \\
\text { de Madrid }\end{array}$ \\
\hline PUENTES, Iván & 2017 & $\begin{array}{l}\text { Las relaciones públicas al servicio de la } \\
\text { comunicación política: los gabinetes de } \\
\text { comunicación en las diputaciones provinciales y } \\
\text { forales }\end{array}$ & $\begin{array}{l}\text { Universidad de } \\
\text { Vigo }\end{array}$ \\
\hline $\begin{array}{l}\text { RAMíreZ, José } \\
\text { María }\end{array}$ & 2017 & $\begin{array}{l}\text { Relaciones públicas de estado: la gestión de } \\
\text { públicos en el reinado de Alfonso XIII (1902- } \\
\text { 1931) a través de sus viajes oficiales al exterior }\end{array}$ & $\begin{array}{l}\text { Universidad de } \\
\text { Sevilla }\end{array}$ \\
\hline RODRíGUEZ, Lucía & 2017 & $\begin{array}{l}\text { Estrategias de gestión de públicos en relaciones } \\
\text { públicas. El ceremonial en el Ayuntamiento de } \\
\text { Sevilla durante el siglo XIX }\end{array}$ & $\begin{array}{l}\text { Universidad de } \\
\text { Sevilla }\end{array}$ \\
\hline Ruíz, Isabel María & 2013 & $\begin{array}{l}\text { Relaciones con los públicos y responsabilidad } \\
\text { social. Estudio de las grandes empresas en } \\
\text { España }\end{array}$ & $\begin{array}{l}\text { Universidad de } \\
\text { Málaga }\end{array}$ \\
\hline SIERRA, Javier & 2007 & $\begin{array}{l}\text { Protocolo: símbolos y comunicación en el siglo } \\
X X I\end{array}$ & $\begin{array}{l}\text { Universidad } \\
\text { Complutense } \\
\text { de Madrid }\end{array}$ \\
\hline SILVA, Carmen & 2016 & $\begin{array}{l}\text { Community managers: relaciones públicas y } \\
\text { comunicación corporativa en la red }\end{array}$ & $\begin{array}{l}\text { Universidad de } \\
\text { Sevilla }\end{array}$ \\
\hline SILVEIRA, Andréia & 2016 & $\begin{array}{l}\text { Relaciones públicas en Brasil y España: un } \\
\text { estudio comparativo entre los campos } \\
\text { profesional y académico (1960-2014) }\end{array}$ & $\begin{array}{l}\text { Universidad de } \\
\text { Málaga }\end{array}$ \\
\hline SMOLAK, Emilia & 2017 & $\begin{array}{l}\text { Relaciones públicas } 2.0 \text { y Think Tanks. Análisis } \\
\text { de Social Media como estrategia de } \\
\text { comunicación }\end{array}$ & $\begin{array}{l}\text { Universidad de } \\
\text { Málaga }\end{array}$ \\
\hline $\begin{array}{l}\text { Solano, Luis } \\
\text { Felipe }\end{array}$ & 2006 & $\begin{array}{l}\text { Fundamentación lógico-formal de la } \\
\text { responsabilidad social corporativa }\end{array}$ & $\begin{array}{l}\text { Universidad } \\
\text { Complutense } \\
\text { de Madrid }\end{array}$ \\
\hline SOLER, Ana & 2017 & $\begin{array}{l}\text { La gestión de la comunicación externa en los } \\
\text { museos andaluces }\end{array}$ & $\begin{array}{l}\text { Universidad de } \\
\text { Málaga }\end{array}$ \\
\hline $\begin{array}{l}\text { SoRIA, María del } \\
\text { Mar }\end{array}$ & 2011 & $\begin{array}{l}\text { La comunicación en las ONGs españolas: la } \\
\text { influencia de Internet en el modelo estratégico } \\
\text { de gestión de públicos }\end{array}$ & $\begin{array}{l}\text { Universidad de } \\
\text { Málaga }\end{array}$ \\
\hline $\begin{array}{l}\text { Soto, Ivette } \\
\text { Maritza }\end{array}$ & 2008 & $\begin{array}{l}\text { Relaciones públicas internacionales: la imagen } \\
\text { de marca país de Puerto Rico y su relación con } \\
\text { el turismo }\end{array}$ & $\begin{array}{l}\text { Universidad de } \\
\text { Málaga }\end{array}$ \\
\hline $\begin{array}{l}\text { TORRES, Rosa } \\
\text { María }\end{array}$ & 2008 & $\begin{array}{l}\text { Aplicación de instrumentos de relaciones } \\
\text { públicas en el ámbito del desarrollo local. } \\
\text { Descripción y posibilidades }\end{array}$ & $\begin{array}{l}\text { Universidad } \\
\text { Autónoma de } \\
\text { Barcelona }\end{array}$ \\
\hline $\begin{array}{l}\text { WALTHER, Amybel } \\
\text { Nila }\end{array}$ & 2007 & $\begin{array}{l}\text { Incidencia de la comunicación y las relaciones } \\
\text { públicas en la formación universitaria limeña }\end{array}$ & $\begin{array}{l}\text { Universidad } \\
\text { San Pablo- } \\
\text { CEU }\end{array}$ \\
\hline
\end{tabular}

Fuente: elaboración propia. 
Como puede observarse en la Tabla 3, la Universidad de Málaga es la sede de presentación de la mayor cantidad de tesis sobre relaciones públicas del periodo 2006-2017, donde lo han hecho uno de cada cuatro doctorandos (9 [23,68\%]). Le siguen la Universidad Complutense de Madrid (5 [13,15\%]); la Universidad Ramon Llull (4 [10,52\%]); la Universidad Autónoma de Barcelona, la Universidad de Sevilla y la Universidad Rovira i Virgili (3 [7,89\%]); y las universidades de Vigo, Rey Juan Carlos, y Camilo José Cela (2 [5,26\%]). En las universidades de Alicante, Navarra, San Pablo-CEU, A Coruña, Carlos III y Cardenal Herrera Oria-CEU se registró únicamente 1 tesis (2,63\%).

Tabla 3. Tesis doctorales sobre relaciones públicas defendidas en universidades españolas en el periodo 2006-2017

\begin{tabular}{lcc}
\hline Universidad & N. $^{\circ}$ de tesis & Porcentaje \\
\hline U. de Málaga & 9 & $23,68 \%$ \\
U. Complutense de Madrid & 5 & $13,15 \%$ \\
U. Ramon Llull & 4 & $10,52 \%$ \\
U. Autónoma de Barcelona & 3 & $7,89 \%$ \\
U. de Sevilla & 3 & $7,89 \%$ \\
U. Rovira i Virgili & 3 & $7,89 \%$ \\
U. de Vigo & 2 & $5,26 \%$ \\
U. Rey Juan Carlos & 2 & $5,26 \%$ \\
U. Camilo José Cela & 2 & $5,26 \%$ \\
U. de Alicante & 1 & $2,63 \%$ \\
U. de Navarra & 1 & $2,63 \%$ \\
U. San Pablo-CEU & 1 & $2,63 \%$ \\
U. de A Coruña & 1 & $2,63 \%$ \\
U. Carlos III & 1 & $2,63 \%$ \\
U. Cardenal Herrera-Oria & 1 & $2,63 \%$ \\
\hline
\end{tabular}

Fuente: elaboración propia.

En cuestión de género, se han contabilizado 20 tesis escritas por mujeres $(52,63 \%)$ y 18 redactadas por hombres $(47,36 \%)$.

El año con mayor producción doctoral del periodo analizado fue 2017 (8 [21,05\%]), seguido por 2011 (5 [13,15\%]); 2014 y 2016 (4 [10,52\%]); 2013 y 2015, 2009 y 2007 (3 [7,89\%]); 2006 y 2008 (2 [5,26\%]); y 2012 $(1[2,63 \%])$. El único año en que no se presentó públicamente ninguna tesis sobre el objeto de estudio fue 2010.

De la muestra final de 38 trabajos defendidos entre 2006 y 2017, 29 $(76,31 \%)$ ostentan el término «relaciones públicas» en el título, ya sea en español, inglés o catalán, al que hay que añadir 1 más $(2,63 \%)$, que incorpora el acrónimo anglosajón «PR», ascendiendo así el total a 30 (78,94\%), es decir, a más de las tres cuartas partes. De las tesis doctorales restantes, 8 (21,05\%) incluyen denominaciones de campo como «relaciones internas», «responsabilidad social corporativa», "gestión de públicos», "comunicación externa», «protocolo y ceremonial» o «relaciones con los públicos». 
Respecto a la cuestión lingüística, se identifican 3 tesis escritas en lengua inglesa $(7,89 \%)$ y $2(5,26 \%)$, en lengua catalana. Las restantes 33 tesis $(86,84 \%)$ fueron escritas en lengua española.

\subsection{Los doctorados sobre Relaciones Públicas en el sistema universitario español (2006-2017)}

La Universidad Complutense de Madrid (Doctorado en Comunicación Audiovisual, Publicidad y Relaciones Públicas); la Universidad Autónoma de Barcelona (Doctorado en Comunicación Estratégica, Publicidad y Relaciones Públicas) —ambas públicas - y la Universidad Camilo José Cela — privada- (Doctorado en Publicidad y Relaciones Públicas, actualmente ya no ofertado), son las únicas del sistema universitario español que ofrecen o han ofrecido programas activos de doctorado que incluyen el término «relaciones públicas» en la denominación de la titulación.

Sin embargo, ante el destacado volumen de tesis sobre relaciones públicas defendidas en la Universidad de Málaga durante este periodo, la investigación analizó, excepcionalmente, el Doctorado Interuniversitario de Comunicación de las universidades de Málaga, Sevilla, Cádiz, Huelva e Internacional de Andalucía — todas ellas públicas-, pese a no contemplar el término de la disciplina en su denominación, ya que el análisis reveló que, aunque no aparecía el concepto «relaciones públicas» en el título del curso, sí contaba con una línea de investigación específica en relaciones públicas desde el curso 2013-2014, razón que estaría en el origen del número de tesis defendidas sobre la materia.

Se estima que estos resultados sufrirán modificaciones sustanciales en el futuro, ya que con respecto a los doctorados españoles en el marco del EEES, regulados por el Real Decreto 99/2011, de 28 de enero, cabe decir que, según Haug (2015: 31-32), en un futuro cercano se prevén nuevos e importantes cambios tendentes a su modernización con relación al nivel del máster,

ya que queda una reforma pendiente en la mayoría de universidades españolas, así como al nivel del sistema en su conjunto [...] A lo largo de la última década tuvo lugar un replanteamiento estratégico fundamental del papel del doctorado en la universidad y la sociedad. Estas reflexiones tienen sus orígenes en el debate sobre la Europa del conocimiento, que enfatiza la importancia de la innovación y la necesidad de reforzar la capacidad competitiva de Europa frente a EE.UU. y a los nuevos poderes del conocimiento de Asia [...] La Asociación de Universidades Europeas (EUA) ha creado en su seno un Consejo por la Educación Doctoral y promueve una nueva visión, nuevos principios organizativos y el desarrollo de un mercado laboral para investigadores en Europa en colaboración con las empresas,

y todo ello basado en los «Diez Principios de Salzburgo», adoptados en 2005, que definen el desarrollo de las nuevas políticas para el doctorado en el territorio europeo, las cuales se estructuran en torno a tres ejes: (1) una mejor 
estructuración en programas de doctorado, agrupados en el marco de escuelas posgraduales o doctorales; (2) la integración en la estrategia global de cada universidad; y (3) la europeización/internacionalización de programas y escuelas doctorales (ibidem: 32 ).

Dichas escuelas posgraduales o doctorales son, fundamentalmente, de dos tipos:

- escuelas posgraduales (graduate schools), que suelen agrupar los másters de investigación y los programas de doctorado de un determinado campo de conocimiento, en el seno de una facultad o en común entre varias facultades, sin tener profesorado propio distinto del de las facultades;

- escuelas doctorales (doctoral schools, research schools), que suelen ser entidades recientes que coordinan y apoyan los doctorados (y posdoctorados) de varias facultades, generalmente sin sustituirse a ellas, pero a veces con un profesorado propio, distinto del de las facultades (ibidem: 33 ).

Es de prever, pues, que conforme las universidades españolas vayan desarrollando e implantando nuevas estrategias globales de doctorados alineadas con las prioridades establecidas a nivel europeo, los objetos de estudio de las tesis doctorales que de ellos se deriven se verán indisolublemente afectados.

\section{Conclusiones}

Las tesis cuyo objeto de estudio ha sido el descriptor "relaciones públicas» han sido proporcionalmente más abundantes en el periodo analizado (20062017) — 38 tesis - que en el anteriormente estudiado por Castillo y Xifra en 2006 (1965-2005) -65 tesis-.

También se advierte, como en el anterior estudio de 2006, que los campos y temas de investigación de la mayoría de trabajos doctorales siguen refiriéndose al análisis de aspectos profesionales de las relaciones públicas y a su dimensión instrumental. Ya se concluía entonces que en el periodo 1965-2005 existía un problema estructural del sistema universitario español a nivel de licenciaturas en Publicidad y Relaciones Públicas, que se sigue manteniendo plenamente vigente en los actuales grados nacidos al amparo de los tres despliegues del Plan de Bolonia y que no es otro que el insuficiente número de asignaturas específicas sobre relaciones públicas, en detrimento de las de publicidad, así como su orientación básicamente técnica y escasamente estratégica (Xifra, 2007; 2015), lo que permite elucubrar sobre los efectos en la elección de los temas de las tesis doctorales, así como sobre su perspectiva prevalentemente técnica.

Los resultados obtenidos también resultan consistentes con Míguez et al. (2016: 19) cuando destacan que la investigación aplicada que posee un carácter general y extrapolable a diferentes ámbitos es la más abundante en la revista Journal of Public Relations Research. 
En esta investigación se ha tenido en cuenta la oferta de los 4 programas de doctorado especializados en Relaciones Públicas, que se observa ínfima en relación con la oferta de los 33 grados de Publicidad y Relaciones Públicas -o equivalentes - ofertados por otras tantas universidades e identificados por Perlado y Saavedra (2017) en el curso 2014-2015. Sin embargo y pese a todo, la producción de las 38 tesis defendidas los últimos doce años, incluso considerando el efecto ANECA (Soriano, 2008), podría considerarse positivamente a tenor de la limitada oferta de programas de doctorado específicos existentes en el sistema universitario español.

En el periodo analizado (2006-2017), debido a la gran proliferación de nuevos grados en Publicidad y Relaciones Públicas - o equivalentes - las presentaciones de tesis se han polarizado, si bien distribuyéndose solo entre algunas pocas universidades. Se comprueba que la Universidad Complutense de Madrid ha ido perdiendo protagonismo conforme pasaban los años y se ha situado en la segunda posición, pasando el testigo del liderazgo a la Universidad de Málaga, donde casi la cuarta parte $(23,68 \%)$ de los doctorandos españoles han preferido defender sus trabajos. Puede atribuirse como causa de esta nueva posición de líder al doctorado malagueño, el más antiguo del país (2006) y único durante un largo periodo de tiempo con una línea de investigación que incluía a las relaciones públicas. Sin embargo, en las otras tres universidades que años después comenzaron a ofertar programas de doctorado en los que figuraría el término «relaciones públicas» en su denominación (Universidad Complutense de Madrid, Universidad Autónoma de Barcelona y Universidad Camilo José Cela), no se aprecian resultados destacables que puedan interpretarse sin ambigüedades en términos de relación causa/efecto en la manufactura de tesis.

En la producción doctoral especializada en relaciones públicas entre 2006 y 2017 tampoco se observa una correspondencia directa con el extraordinario crecimiento de la oferta de estudios de grado de Publicidad y Relaciones Públicas que se origina coincidiendo con la entrada al nuevo milenio, que pasó de 19 en el año 2000 (Tilson y Pérez, 2003) a 33 en 2014 (Perlado y Saavedra, 2017). Desde una perspectiva comparativa, la obligatoriedad histórica, anterior al EEES, de impartir de manera conjunta los contenidos de publicidad y de relaciones públicas se ha constatado muy perjudicial para la segunda disciplina en ambos periodos. Como se ha señalado anteriormente, la presencia de asignaturas específicas de relaciones públicas estaba muy por debajo de las de publicidad en el periodo analizado por Castillo y Xifra (2006: 155), siendo además de marcado carácter táctico (Xifra, 2006). Casi una década después, ya avanzado el despliegue del EEES, la situación no había mejorado sustancialmente (Xifra, 2015). No es de extrañar, pues, que no habiéndose producido cambios en este sentido entre el primer y el segundo periodo analizados, tampoco se observen modificaciones en las temáticas abordadas en la producción doctoral española, que siguen analizando mayoritariamente cuestiones relacionadas con la praxis profesional y a su aspecto instrumental (investigación aplicada), en detrimento de las aproximaciones 
teóricas (investigación básica). Esta circunstancia ha provocado que se consolide cada vez más el problema estructural del que adolece la enseñanza de las relaciones públicas en la universidad española desde sus inicios - y en especial en el nivel del posgrado- que, al ser de carácter sistémico, se ha convertido en crónico.

La progresiva y próxima incorporación de las universidades españolas a los «Diez Principios de Salzburgo» (2005), que afectarán a la modernización de los actuales másters oficiales y doctorados en vigor, permiten especular acerca de un probable cambio en la orientación de los objetos de estudio de la producción de tesis doctorales asociada a estos. Será interesante, entonces, verificar o refutar si esta predicción cristaliza en realidad, y analizar en profundidad sus consecuencias en el ámbito específico de las relaciones públicas, cuestión que nos proponemos abordar llegado ese momento.

No es objeto de este estudio relacionar las tesis doctorales resultantes con los artículos derivados de las mismas y publicados en revistas especializadas, investigación que, dado su interés, nos proponemos desarrollar en próximas investigaciones.

Esta investigación es un encargo de transferencia de conocimiento de la Asociación de Directivos de Comunicación Dircom Catalunya, en el marco del Observatorio de la Comunicación de la Cátedra Dircom.Cat de su Comisión de Formación y Docencia.

\section{Referencias bibliográficas}

Agudelo, D.; Bretón-López, J.; Ortiz-Recio, G.; PovedA-Vera, J.; TeVA, I.; VALOR-SEGURA, I. y ViCO, C. (2003). «Análisis de la productividad científica de la Psicología espańola a través de las tesis doctorales». Psicothema, 15 (4), 595609.

Botan, C. H. y Hazleton, V. (2006). «Public relations in a new age». En: C. H. Botan y V. Hazleton, Public Relations Theory II (1-18). Mahwah, NJ: Lawrence Erlbaum.

BOYNTON, L. (2006). «What we value: A Delphi study to identify key values that guide ethical decision-making in Public Relations». Public Relations Review, 32 (4), 325-330.

CAMPS, D. (2008). «Limitaciones de los indicadores bibliométricos en la evaluación de la actividad científica biomédica». Colombia Médica, 39 (1), 74-79.

Castillo-Esparcia, A.; Rubio-Moraga, A. y Almansa-Martínez, A. (2012). «La Investigación en comunicación. Análisis bibliométrico de las revistas de mayor impacto del ISI». Revista Latina de Comunicación Social, 67, 248-270.

Castillo-Esparcia, A.; Ruiz-Mora, I. y Álvarez-Nobell, A. (2016). «Procesos de institucionalización de la investigación sobre comunicación: la reconfiguración de la estructura de la oferta de los estudios de doctorado en Comunicación en España». En: B. DíAz NOstY y R. de FrUTOS (coords.). Rumbos de la investigación en España sobre Comunicación (703-718). III Congreso Nacional de Metodología de la Investigación en Comunicación. Málaga, 12-13 noviembre de 2015. 
CASTillo, A. y Xifra, J. (2006). «Investigación bibliométrica de las tesis doctorales españolas sobre relaciones públicas». Anàlisi. Quaderns de Comunicació i Cultura, 34, 141-161.

Castillo, A.; Álvarez, A. y MuÑiz, J. A. (2013). «EEES y la formación en comunicación en España. La estructura de la oferta de postgrado». Estudios sobre el Mensaje Periodístico, 19, especial marzo, 99-110.

Cuenca, J.; Compte, M.; Matilla, K. y Hernández, S. (2017). «Un análisis de los másters universitarios españoles especializados en Relaciones Públicas y Comunicación Corporativa, curso 2016-2017, desde la perspectiva de las Relaciones Públicas». Obra Digital, 13, 37-57.

FERGUSON, M. A. (1984, agosto). Building theory in public relations: Interorganizational relationships as public relations paradigm. Comunicación presentada en la Conferencia Anual de la Association for Education in Journalism and Mass Communication, Gainesville, FL.

Fuentes Pujol, E. y ARguimbau Vivó, LL. (2010). «Las tesis doctorales en España (1997-2008): análisis, estadísticas y repositorios cooperativos». Revista Española de Documentación Cientifica, 33 (1), 63-89.

GARCÍA LEIVA, T. (2011). «La digitalización de la comunicación y la cultura». Telos, Cuadernos de Comunicación e Innovación, 83.

Gonzalez De Dios, J.; Moya, M. y Mateos Hernandez, M. A. (1997). Indicadores bibliométricos: características y limitaciones en el análisis de la actividad científica. Anales Españoles de Pediatría, 47 (3), 235-244.

HAUG, G. E. (2015). Grados y másteres en España y el Espacio Europeo de Educación Superior (EEES). Madrid: CRUE-Universidades Españolas, Colección Monografías.

Maltras-BArba, B. (2003). Indicadores bibliométricos. Fundamentos y aplicación al análisis de la ciencia. Gijón: Trea.

Matilla, K.; Cuenca-Fontbona, J. y Marca-Francés, G. (2014). «Ya me he graduado en Relaciones Públicas. Y, ahora, ¿qué? Una mirada a la formación de postgrado oficial en Espańa (2013-2014)». En: J. FombOnA CADAVIECO y D. CaldeVilla DomíngueZ. Nuevas formulaciones de los contenidos docentes (capítulo 32, 373-383). Madrid: McGraw Hill Education.

MCELREATH, M. P. (1980). Priority research questions for public relations for the 1980s. Nueva York: Foundation for Public Relations Research and Education.

- (1989). "Priority research questions in the field of public relations for the 1990s: trends over the past ten years and predictions for the future». Meeting of the Speech Communication Association, San Francisco, CA.

MCELREATH, M. P. y BlampHIN, J. M. (1994). «Partial answers to priority research questions - and gaps- found in the Public Relations Society of America's body of knowledge». Journal of Public Relations Research, 6 (2), 69-103.

Merlo VeGA, J. A. y Sorli Rojo, A. (2002). «Bases de datos y recursos en Internet sobre tesis doctorales». Revista Española de Documentación Científica, 25 (1), 95-106.

Miguel Alonso, A. (2000). «Aportaciones al estudio de la literatura gris universitaria: La evolución de la tesis doctoral en España». I Congreso Universitario de Ciencias de la Documentación: Teoría, historia y metodología de las ciencias de la documentación, 645-651. Madrid: Universidad Complutense de Madrid.

MígueZ-GonzÁlez, M. I.; CorbaCho-Valencia, J. M. y BaAmonde-Silva, X. M. (2016). «Tendencias de investigación sobre relaciones públicas en revistas 
internacionales: el caso de Journal of Public Relations Research, 2012-2014». Revista Internacional de Relaciones Públicas, 6 (12), 5-24.

Ministerio De EdUCACión (2011). Real Decreto 99/2011, de 28 de enero, por el que se regulan las enseñanzas oficiales de doctorado. $B O E n^{\circ} 35,10$ de febrero de 2011, Sec. 1, pág. 13.909.

Ministerio De Educación, Cultura y Deporte (MECD) (2017). «Avance de la Estadística de estudiantes. Curso 2016-2017» [datos provisionales]. En: Sistema integrado de información universitaria. Madrid: MECD.

Moralejo Álvarez, M. R. (2000). "Las tesis doctorales de las universidades espańolas: control bibliográfico y acceso». Revista General de Información y Documentación, 10 (1), 235-243.

Moreno-Fernández, O. y Moreno-Crespo, P. (2016). «Análisis bibliométrico de las tesis doctorales españolas indexadas con el descriptor 'Sector de la educación’ (1976/2014)». Revista Española de Documentación Cientifica, 39 (3), e146. <http://dx.doi.org/10.3989/redc.2016.3.1331>

OrerA-OrerA, L. (2003). «Bibliotecas digitales de tesis doctorales: Metodología para su planificación». Boletín de la Asociación Andaluza de Bibliotecarios, 72 , 55-72.

PeŃAfiel, C.; TORres, E. y IzQuierdo, P. (2016). «Percepción cualitativa de gestores universitarios de investigación en Comunicación a través de un Philips 66». En: J. HeRrero y C. MATEOS (coords.). Del verbo al bit (790-810). Tenerife: Universidad de La Laguna, Cuadernos Artesanos de Comunicación \#116.

Perlado-Lamo De Espinosa, M. y SaAvedra-Llamas, M. (2017). Los estudios universitarios especializados en Comunicación en España. Barcelona: UOC, Colección Dircom, n. ${ }^{\circ} 8$.

RAUPP, J. y VAN RULER, B. (2006). «Trends in public relations and communication management research: a comparison between Germany and The Netherlands». Journal of Communication Management, 10 (1), 18-26.

Sallot, L. M.; LyOn, L. J.; ACOSTA-Alzuru, C. y JONES, K. O. (2003). «From aardvark to zebra: A new millennium analysis of theory development in public relations academic journals». Journal of Public Relations Research, 15 (1), 27-90.

SYNNOTT, G. y MCKIE, D. (1997). «International issues in PR: researching research and prioritizing priorities». Journal of Public Relations Research, 9 (4), 259-282.

Tato García, M.; TATo Jiménez, J. L. y Castillo Díaz, A. (2014). «La investigación en comunicación en España: estado de la cuestión». Revista RAE-IC, 1 (1), 129-139.

Tilson, D. J. y PÉREZ, P. S. (2003). «Public relations and the new golden age of Spain: a confluence of democracy, economic development and the media». Public Relations Review, 29 (2), 125-143.

VAn Ruler, B.; VerČIČ, D.; BÜTSCHI, G. y Flodin, B. (2004). «A First Look for Parameters of Public Relations in Europe». Journal of Public Relations Research, 16 (1), 35-63.

VV. AA. (2005). Los diez principios de Salzburgo. Seminario Bolonia "Doctoral Programmes for the European Knowledge Society», Salzburgo. $<$ https://www.eua.eu/ component/attachments/attachments.html?task=attachment\&id=1881>.

WAKEFIELD, R. (2000). «Preliminary Delphi research on international public relations programming: initial data supports application of certain generic/specific concepts». En: D. MOss, D. VERČIČ, y G. WARnABY (eds.). Perspectives on public relations research (179-208). Londres: Routledge. 
WATSON, T. (2008a). «Public relations research priorities: a Delphi Study». Journal of Communication Management, 12 (2), 104-123.

- (2008b). «Research Priorities for Public Relations: The Asia Pacific insights from an international Delphi study». PRism 5 (1-2).

White, J. y BLAMPHIN, J. (1994). Priorities for research into public relations practice in the United Kingdom. Londres: City University Business School / Rapier Research.

XIFRA, J. (2007). «Undergraduate public relations education in Spain: Endangered species?». Public Relations Review, 33 (2), 206-213.

- (2015). «La 'enseñanza' universitaria de la comunicación corporativa en Cataluña». En: K. Matilla (ed.). Historia de la Comunicación Corporativa en Cataluña (147-199). Barcelona: UOC.

XifrA, J. y CASTILLO, A. (2006). "Forty years of doctoral public relations research in Spain: A quantitative study of dissertation contribution to theory development». Public Relations Review, 32, 302-308. 
